\title{
Protocolo de vigilancia epidemiológica para la prevención de lesiones derivadas del entrenamiento físico-militar
}

https://doi.org/10.21830/9789585284814.13

\author{
Yenny Paola Argüello Gutiérrez", Laura Elizabeth Castro Jiménez², \\ Paula Janyn Melo Buitrago ${ }^{3}$
}

\section{Resumen}

Introducción: el entrenamiento físico militar conlleva altas exigencias físicas en los sujetos, entre ellas, por ejemplo, el tiempo de concentración, por las horas de entrenamiento y por la densidad de las actividades, lo que se asocia, inevitablemente, con la aparición de lesiones, las cuales pueden truncar la vida militar de los afectados. Objetivo: proponer un sistema de vigilancia epidemiológica para la prevención de lesiones derivadas del entrenamiento físico militar. Metodología: estudio con alcance exploratorio, diseńo longitudinal-prospectivo de tres meses de seguimiento, en el cual, por medio de la revisión de la literatura, se diseńaron y validaron las fichas de reporte y seguimiento de "Evento epidemiológico", posterior a su aplicación a los cadetes de primer semestre de Relaciones Internacionales e Ingeniería Civil que presentaban algún tipo de lesión. Resultados: diseño de un sistema de vigilancia epidemiológica como estrategia de control y prevención de lesiones derivadas del entrenamiento físico militar. Conclusiones: la vigilancia epidemiológica permitirá la disminución de las lesiones asociadas al entrenamiento militar, gracias a la toma de decisiones oportunas sobre los casos probables, sospechosos y confirmados de lesiones que se determinen por medio del sistema diseñado.

Palabras clave: entrenamiento físico militar; lesiones; sistema de vigilancia epidemiológico.

1 Fisioterapeuta. Magister en Fisiología. Docente Investigadora Grupo Cuerpo, Sujeto y Educación. Universidad Santo Tomás. Contacto: yenniarguello@usantotomas.edu.co. Orcid: https://orcid.org/0000-0001$8335-4936$

2 Fisioterapeuta. Magister en Salud Pública. Doctorando en Humanidades, Humanismo y Persona. Docente Investigadora Grupo Cuerpo, Sujeto y Educación. Universidad Santo Tomás. Contacto: laura.castro@usantotomas.edu.co. Orcid: https://orcid.org/0000-0001-5166-8084

3 Licenciada en Educación Física, Especialista en Docencia Universitaria, Magister Educación con énfasis en Pedagogía del Entrenamiento Deportivo. Coordinadora de Investigaciones Facultad de Educación Física militar. Grupo de investigación RENFIMIL - Escuela Militar de Cadetes General José María Córdova. Contacto: paula.melo@esmic.edu.co. Orcid: https://orcid.org/0000-0002-3492-7985 


\section{Introducción}

La conformación de ejércitos, y con ello la formación integral de futuros oficiales, se convierte en un ejercicio de soberanía para varios países. Dentro de la formación integral de los oficiales se contempla el entrenamiento militar clásico y una rutina académica universitaria en combinación con la formación militar (Martínez, 2011; Rodríguez, Valenzuela, Velasco, Castro \& Melo, 2016). Es así como realizan dos tareas combinadas: un intenso entrenamiento militar que conlleva un constante reto físico (Olmedilla, Ortega, Prieto \& Blas, 2009; Heinrich, Spencer, Fehl \& Poston, 2012) y una rutina académica universitaria que implica esfuerzo, dedicación y horas de estudio, tal como es el caso de la Escuela Militar de Cadetes "General José María Córdova”.

La literatura muestra de forma consistente cómo el entrenamiento militar conlleva lesiones de origen musculoesquelético, dada la necesidad de que los reclutas logren altos niveles de aptitud física en cortos periodos de tiempo (Mohammadi, Azma, Naseh, Emadifard \& Etemadi, 2013). Las cinco lesiones comúnmente más reportadas en la población militar son: síndrome de estrés o uso excesivo, distensiones musculares, esguinces de cuello de pie, lesiones de rodilla por uso excesivo y fracturas por estrés (Jones et al., 1993; Rodríguez et al., 2016; Ríos, Castro \& Melo, 2016), dichas lesiones conllevan a procesos de recuperación prolongados en la mayoría de los casos e incluso pueden separar de manera definitiva al cadete de la vida militar, esto, sin mencionar los costos que implican los tratamientos y aquellos casos de lesiones reiterativas que aumentan de manera significativa los presupuestos en salud. De esta manera, la presencia de lesiones se vuelve una carga para el Ejército Nacional, por lo que implica para la salud y la buena condición física de sus soldados, así como para el financiamiento del sistema general de seguridad social.

Para mitigar los problemas en salud de la población se han planteado diferentes estrategias, como la generación de programas de promoción de la salud, prevención de factores de riesgo y de enfermedad, así como el diseño y la implementación de sistemas de vigilancia epidemiológica. Esta última implica la recolección de información y la utilización de la misma para conseguir un determinado objetivo, cambiando las circunstancias que favorecen 
la aparición de la situación problema o manteniendo aquellas que controlan dicha situación (Restrepo, 2013; Liabsuetrakul, Prappre, Pairot, Oumudee \& Islam, 2017).

$\mathrm{Al}$ revisar la literatura se evidencia la importancia de los sistemas de vigilancia epidemiológica (Pujol \& Limón, 2013) y la carencia de estos sistemas al interior del Ejército Nacional como forma de monitorear la salud de sus soldados y mitigar factores de riesgo existentes en el ejercicio diario. Se ha reportado muy poca información acerca de programas de prevención sobre lesiones musculo-esqueléticas y sistemas de vigilancia epidemiológica en militares; las investigaciones que se reportan alrededor del tema están relacionadas con intervenciones psicológicas (Ghahramanlou-Holloway et al., 2014; Luxton et al., 2014), salud oral (Spalj, Spalj, Ivankovic \& Plancak, 2014) y factores de riesgo asociados (Brandon et al., 2014), siendo importante resaltar que no se encontró información propia de nuestro país.

En este sentido, la presente investigación plantea el diseño de un sistema de vigilancia epidemiológica en lesiones osteomusculares, dado que, según datos del Ministerio del Trabajo, desde el año 2009 son la principal enfermedad de origen laboral y cuentan con una incidencia de 11.6 casos por cada 10.000 trabajadores (Ministerio de Trabajo, 2013). El sistema tiene como objetivo articular la vigilancia de efectos y de factores de riesgo (enfoque preventivo), para evitar que se desarrollen signos y síntomas, efectos o condiciones no deseadas en la población militar y, por consiguiente, las enfermedades derivadas de su rol laboral (Botero, 2016). De acuerdo con lo mencionado por la Organización Internacional del Trabajo (OIT), la vigilancia de la salud debería ser parte de todos los sistemas de salud para mejorar las condiciones de calidad de vida de toda la población (Organización Internacional del Trabajo, 1998).

\section{Métodos}

Estudio con alcance exploratorio, diseño longitudinal-prospectivo de tres meses de seguimiento, que se desarrolló en tres fases.

Fase 1: determinar las características del sistema de vigilancia epidemiológica, en la cual se realizó una revisión de la literatura para generar los elementos 
constitutivos, así como la propuesta de las fichas de reporte y seguimiento de los eventos a reportar. La información recolectada con la ecuación de búsqueda se almacenó en una base datos en Excel, posteriormente se clasificó de acuerdo con las siguientes tres categorías: categoría 1, presenta información sobre sistemas de vigilancia epidemiológica en lesiones osteomusculares y factores de riesgo en población militar; categoría 2 , contienen información importante pero no en la población de estudio o sobre el efecto que se vigilará; y categoría 3 , no contiene información importante para la investigación. Con dicha categorización se procedió a analizar la información y establecer categorías relevantes para el diseño de las fichas de reporte y seguimiento del evento. Luego se continuó con la evaluación de validez de las fichas de seguimiento a través de juicios de expertos, así, se contó con la participación de un número de expertos impar (tres en este caso), que tenían un nivel de formación mínimo de maestría o formación doctoral, con experiencia en el tema de interés. Los expertos diligenciaron un formato de evaluación que posteriormente se sistematizó para determinar el acuerdo en las respuestas de los jueces y poder establecer los ajustes que debían ser incorporados en las fichas. Luego de la incorporación de los ajustes, las fichas quedaron validadas.

La Fase 2 consistió en la aplicación de los formatos de reporte y seguimiento que, una vez validados, a modo de estudio piloto, fueron aplicados a los estudiantes de primer nivel (Compañía Voltígeros, Aulas de primer semestre de Ingeniería Civil y Relaciones Internacionales). Las fichas se aplicaron a los estudiantes que presentaban alguna lesión músculo-esquelética y se hizo postea riormente seguimiento por la cantidad de semanas que la lesión ameritara. Las fichas fueron almacenadas y custodiadas por el grupo de investigadoras y ninguna persona fuera de ellas tuvo acceso a los datos. Con dicha información, al finalizar los tres meses del estudio piloto, se realizó un análisis descriptivo en lo referente a la incidencia de lesiones y tipos de lesión, mecanismos de lesión y procesos de rehabilitación, es decir, cada uno de los constructos contenidos en las fichas.

Finalmente, la Fase 3 se direccionó a la construcción de la propuesta del sistema de vigilancia definitivo. 


\section{Resultados}

Acogiendo la "Guía Metodológica para el desarrollo de protocolos de vigilancia de interés en salud pública en Colombia” — la cual reglamenta el Sistema de Vigilancia en Salud Pública (Sivigila)—, propuesta por el Ministerio de Salud y Protección Social en colaboración con la Universidad del Valle en el año 2013, el Sistema de Vigilancia Epidemiológica para la Escuela Militar de Cadetes "General José María Córdova” cuenta con la siguiente estructura (ver Figura 1):

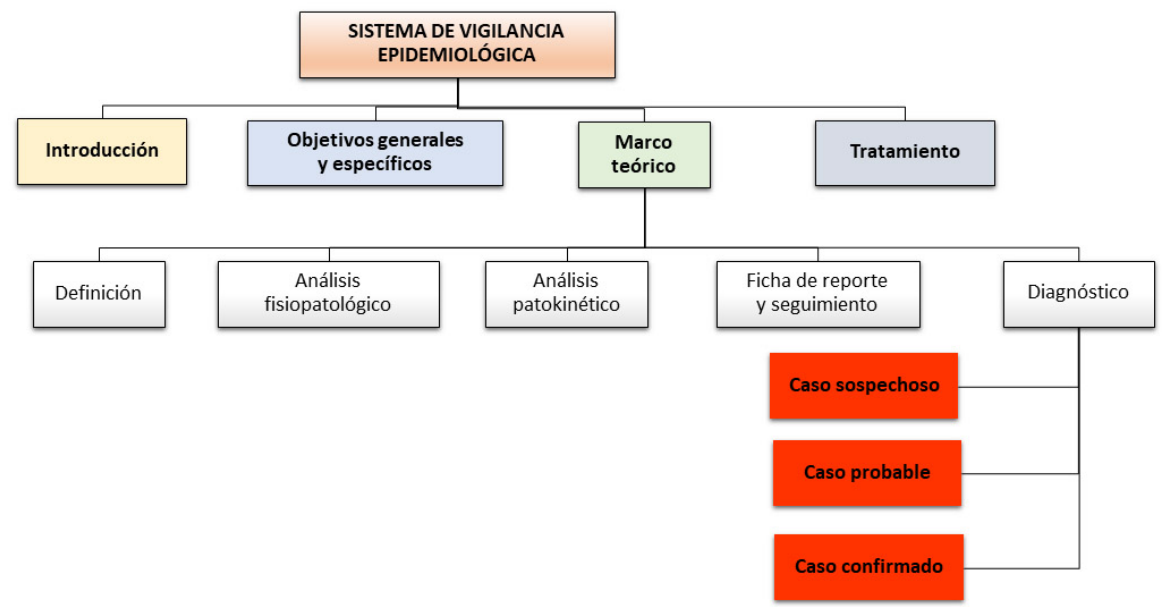

Figura 1. Estructura del Sistema de Vigilancia Epidemiológica Escuela Militar de Cadetes "General José María Córdova".

Fuente: elaboración propia.

La Figura 1 puede desglosarse más detalladamente de la siguiente manera. La "Introducción" contextualiza el problema abordado, que en este caso trata acerca las lesiones derivadas del entrenamiento físico militar. Los "Objetivos general y específicos" giran en torno a la identificación de lesiones derivadas del entrenamiento físico militar de la Escuela Militar de Cadetes "General José María Córdova”, con el fin de adoptar medidas preventivas. El "Marco Teórico”, en el cual se detallan los eventos a reportar, se centra en las lesiones más prevalentes en la población objeto de la vigilancia epidemiológica, que 
para la Escuela Militar de Cadetes fueron: esguince de cuello de pie y periostitis. De cada evento (lesión) se describe su:

- Definición: descripción fisiopatológica y ubicación corporal detallada, si aplica se deben consignar los diferentes estadios o clasificación según gravedad de la lesión.

- Análisis fisiopatológico: se enlistan las causas y factores de riesgo de la patología, junto con su explicación por fases de lesión.

- Análisis patokinético: esquema gráfico (mapa conceptual) que incluye la definición de la patología, causas y factores de riesgo asociados, clasificación de la patología, e implicación de esta patología en mínimo 3 y máximo 5 categorías del movimiento.

- Ficha de reporte de evento y seguimiento de evento: la primera incluye las categorías: datos sociodemográficos, tipo de lesión y mecanismo de lesión; por su parte, la ficha de seguimiento incluye: datos sociodemográficos, tipo de lesión y proceso de rehabilitación.
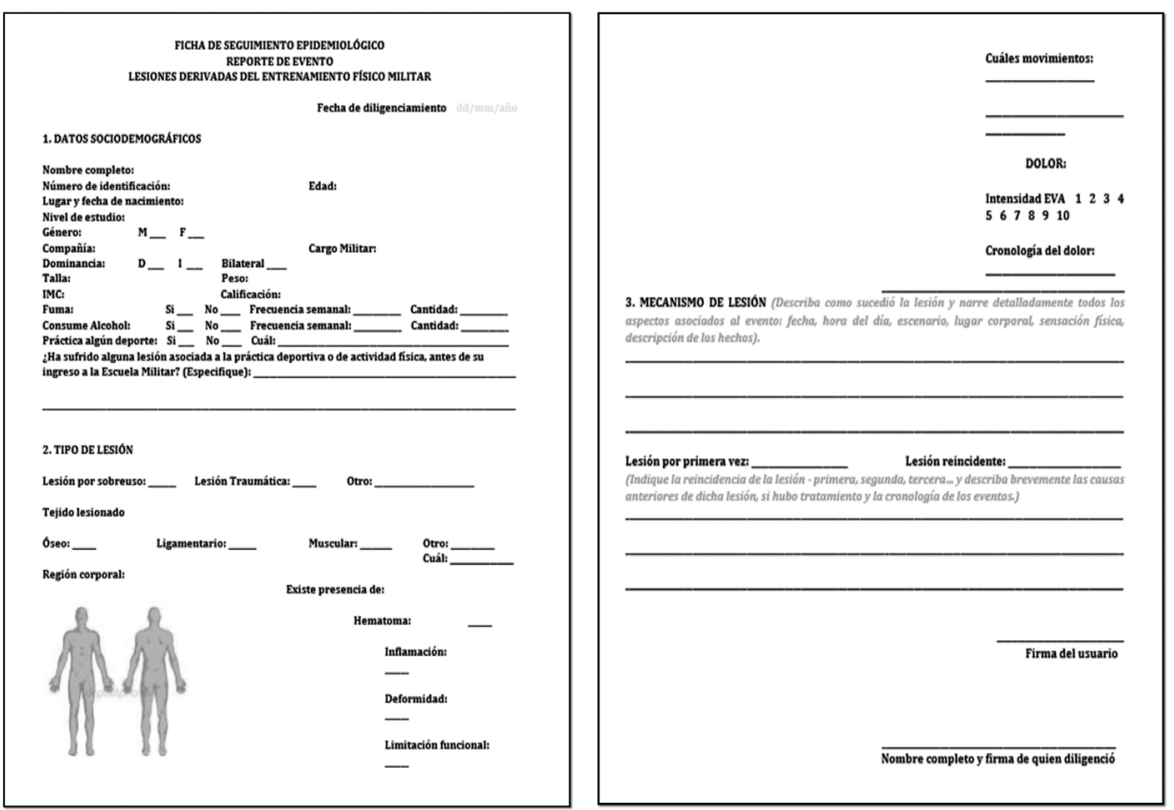

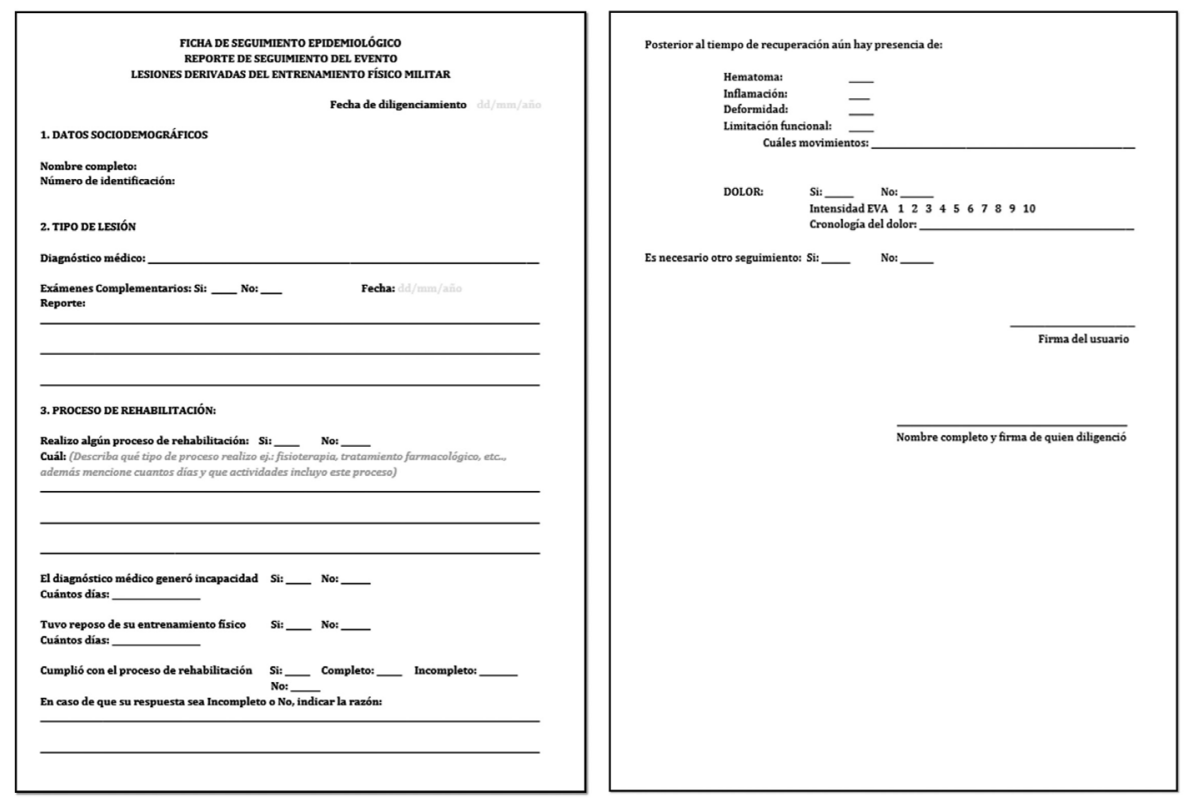

Figura 2. Ficha de reporte de evento y seguimiento de evento.

Fuente: elaboración propia.

- Diagnóstico: A partir de la denominación de la patología, se detallan:

a. Signos y síntomas.

b. Signos patognomónicos.

c. Pruebas específicas.

d. Exámenes paraclínicos.

Para poder concluir con alguno de los siguientes resultados: caso probable, aquel que presenta los ítems a, b y c, pero no se tienen exámenes paraclínicos de confirmación del diagnóstico; caso sospechoso, aquel que presenta los ítems a y b, pero las pruebas específicas no se han realizado o son negativas y además no se cuenta con exámenes paraclínicos; caso confirmado, aquel que presenta los ítems a, b, c y d; y, finalmente, tratamiento propuesto desde el área de fisioterapia. Se plantea de forma resumida el tratamiento fisioterapéutico.

Así mismo, el Sistema de Vigilancia Epidemiológica fue aplicado a los cadetes incluidos en el estudio por medio del diligenciamiento de las fichas de reporte y de seguimiento, y en concordancia con las características del sistema 
que ya fueron descritas. Durante tres meses se aplicaron 10 fichas de reporte de evento ( 3 pertenecientes al grupo de control y 7 al de intervención) y 9 fichas de seguimiento de evento ( 4 cadetes del grupo control y 5 del grupo de intervención).

Los Datos Sociodemográficos incluidos en la ficha de reporte mostraron los siguientes resultados (ver Tabla 1).

Tabla 1. Resultados datos sociodemográficos fichas de reporte de evento

\begin{tabular}{lccc}
\hline \multicolumn{1}{c}{ Variable } & Promedio & Moda & Mediana \\
\hline Edad (años) & 18,33 & 18 & 18 \\
Talla (centímetros) & 176,4 & 173 & 175,5 \\
Peso (Kilogramos) & 69,6 & 72 & 69 \\
IMC & 22,3 & 24 & 21,9 \\
\hline
\end{tabular}

Fuente: elaboración propia.

Con estos datos se evidencia que, en promedio, el grupo de cadetes se encuentra con un IMC de 22,3, que según la clasificación de la OMS es un dato de Peso Normal (Servicio de Vigilancia Epidemiológica, Centro Nacional de Epidemiología. Instituto de Salud Carlos III); por otra parte, el 80\% cuenta con dominancia manual derecha, el 10\% izquierda y el restante $10 \%$ bilateral. Dentro del grupo ningún cadete fuma y el 30\% refiere consumir alcohol ocasionalmente ( $1 \mathrm{vez}$ al semestre o al año); así mismo, ninguno de los cadetes presenta antecedentes de lesiones antes del ingreso a la Esmic.

Con relación a la categoría tipo de lesión se cuenta con un $80 \%$ de lesiones por sobreuso, $10 \%$ de tipo traumático y el restante $10 \%$ de tipo vascular. El tejido más lesionado según los resultados es el óseo, con un 60\%, seguido de un $20 \%$ en la categoría "Otro", con referencia a los tejidos vascular y tendinoso, y un $10 \%$ para cada uno los tejidos muscular y ligamentario.

La región corporal más afectada son las piernas, con un 40\%, seguidas por las rodillas, con un $20 \%$. Las otras regiones que tan solo cuentan con una referencia son: hombro, pies, cuello de pie y muslo; en este aspecto la localización es un $40 \%$ a nivel bilateral, un $40 \%$ en el hemicuerpo izquierdo y el restante $20 \%$ en el lado derecho. 
Con relación a la presencia de dolor, 9 de los encuestados dieron respuesta afirmativa; se encuentra una intensidad promedio de 5/10 según la EVA, con un máximo de dolor referido en 8/10 y mínimos de 1/10. La cronología del dolor fue reportada por el $50 \%$ de los encuestados, con respuesta de dolores de 2 días y otros de 15 días de presencia.

En cuanto a la presencia de signos y síntomas, lo más representativo, con un $80 \%$, es que los cadetes refieren tener inflamación en la región corporal afectada, un 10\% hematoma y el restante 10\% limitación funcional. Sin embargo, de manera concomitante, 4 cadetes presentan inflamación y limitación funcional.

En los encuestados, el $80 \%$ refiere estar lesionado por primera vez y el $20 \%$ refiere lesiones reincidentes. En cuanto a la descripción de los mecanismos de lesión, se encuentra de manera reiterada que el dolor aparece durante o después de las sesiones de entrenamiento físico militar.

En cuanto a las 9 fichas de seguimiento de evento, estas mostraron los siguientes resultados: con relación a la categoría tipo de lesión se cuenta con un $78 \%$ de cadetes que no tienen un diagnóstico médico a pesar de presentar signos y síntomas como inflamación, reportados en la "Ficha de reporte de evento"; el restante $22 \%$ presenta diagnóstico de esguince de cuello de pie y periostitis tibial; este mismo porcentaje cumplió y completó un proceso de rehabilitación tipo fisioterapia. Ninguno de los cadetes cuenta con exámenes paraclínicos; por otro lado, se reporta tan solo en dos cadetes un proceso de incapacidad médica que no superó los 5 días y reposo del entrenamiento militar que para uno de ellos fue de 10 días. Es importante referir que además de estos dos cadetes, uno que no se encontraba incluido en un proceso de rehabilitación, debido a su situación dolorosa, tuvo 5 días de incapacidad y por ende de reposo de su entrenamiento físico militar.

De otro lado, dos cadetes manejaron su proceso doloroso con cremas analgésicas que adquirieron bajo voluntad, las cuales aplicaron todas las noches por lapsos de hasta una semana. Por último, de los dos cadetes incluidos en el proceso de rehabilitación fisioterapéutica, uno de ellos tuvo resolución completa de su lesión y el otro continuaba con un dolor de 2/10, según EVA asociada a la reincorporación al entrenamiento físico militar. 


\section{Discusión}

El Sistema de Vigilancia Epidemiológica diseñado para la prevención de lesiones derivadas del entrenamiento físico militar en el Escuela Militar de Cadetes "General José María Córdova”, se constituye en una estrategia que, a largo plazo, propende por la disminución en las tasas de prevalencia de lesiones en dicha población, que tiene factores de riesgo asociados a su actividad militar habitual; esto, a través de la implementación de unas fichas de reporte y seguimiento de eventos (lesiones), que permitirán reconocer las lesiones, mecanismos de lesión y la vinculación de los cadetes a procesos de rehabilitación que les permitan un reintegro oportuno y adecuado a sus actividades militares. Este diseño se obtuvo gracias a la aplicación (estudio piloto) desarrollada y la revisión de la literatura científica, tal como lo hizo Botero (2016) en un ámbito laboral donde logró, a través de su propuesta, identificar los factores de riesgo biomecánico e implementar una herramienta que da pautas de control y prevención de enfermedades laborales.

El entrenamiento físico militar tiene varios factores de sobrecarga mecánica tipificados (número de horas de entrenamiento, indumentaria utilizada, nivel de condición física previa al ámbito militar, entre otras) (Araújo, Muniz, Sanches, Turi \& Monteiro, 2017), lo que explica la presencia de lesiones generalmente musculo-esqueléticas y con muy malos procesos de recuperación. Ahora bien, en este estudio se encontró que existe un factor comportamental en los cadetes que incide de manera especial en la presencia y cronicidad de sus lesiones, consiste en que no reportan sus dolores o molestias físicas y mucho menos asisten a un servicio médico o proceso de rehabilitación, ya que esto implica días de reposo o incapacidad médica, lo que los aleja por días de su entrenamiento militar, el cual es necesario para la consecución de logros físicos solicitados a los cadetes al final del semestre que, de no ser conseguidos, puede significar el retiro de la Escuela Militar, tal como lo plantean varios autores (Hauret et al., 2015).

Derivado del punto anterior, se hace indispensable la concientización sobre la importancia del reporte de las lesiones, basado en la salud y calidad de vida de los cadetes, por parte de los oficiales y superiores de la Escuela, para 
contar con un ambiente propicio para la notificación, divulgación y manejo de estas situaciones.

Las lesiones reportadas por los militares, en concordancia con lo reportado por Gómez (2017), continúa siendo la periostitis tibial, en el primer lugar; condición que debe ser analizada a profundidad a través de este sistema y que necesariamente implica incorporar los cambios necesarios para minimizar al máximo los factores de riesgo en los cadetes.

El sistema de vigilancia diseñado puede ser aplicado a toda la comunidad de cadetes, se construyó de tal manera que pueda ser aplicado por cualtı quier profesional del área de la salud o que esté vinculado al dispensario de la Escuela. De manera muy especial, se debe vincular a su implementación a aquellos profesores o militares encargados de la dirección o control del entrenamiento físico, por ser un momento crucial para el reporte de las lesiones. En los sistemas o protocolos de vigilancia encontrados se establece el reporte desde el ámbito local hasta nacional, bien sea porque resulta necesario o porque es solicitado por instancias competentes (Fadúl, 2016).

Por último, este proyecto se convierte en un insumo inicial muy importante para la Escuela Militar en lo que atañe a la prevención de lesiones, lo que redundará en la disminución del número de casos reportados cada año.

\section{Referencias}

Botero, P. (2016). Sistema de vigilancia de factores de riesgo biomecánico (Tesis de posgrado). Universidad de Antioquia.

Brandon, T., Klesges, R., Ebbert, J., Talcott, G., Thomas, F., Leroy, K., Richey, P., \& Colvin,

L. (2014). Preventing smoking initiation or relapse following 8.5 weeks of involuntary smoking abstinence in basic military training: trial design, interventions, and baseline data. Contemporary clinical trials, 38(1), 28-36.

Castro, A., \& Díaz, J. (2002). Objetivos de vida y satisfacción vital en adolescentes españoles y argentinos. Psicothema, 14(1), 112-117.

Fadúl, S. (2016). Protocolo de vigilancia en salud pública. LEPRA. Instituto Nacional de Salud.

Ghahramanlou-Holloway, M., Brown, G., Currier, G., Brenner, L., Knox, K., Grammer, G., Carreno-Ponce., \& Stanley, B. (2014). Safety planning for military (safe mil): rationale, design, and safety considerations of a randomized controlled trial to reduce suicide risk among psychiatric inpatients. Contemporary clinical trials, 39(1), 113-123. 
Gómez, S. (2017). Efectividad de las ondas de choque extracorpóreas focalizadas en el tratamiento del sindrome de estrés tibial medial (Tesis de posgrado). Universidad Complutense de Madrid.

Hauret, K., Bedno, S., Loringer, K., Kao, T., Mallon, T., \& Jones, B. (2015). Epidemiology of exercise- and sports-related injuries in a population of young, physically active adults: a survey of military servicemembers. The American journal of sports medicine, 43(11), 2645-53.

Heinrich, K., Spencer, V., Fehl, N., \& Poston, W. (2012). Mission essential fitness: comparison of functional circuit training to traditional Army physical training for active duty military. Military medicine: international journal of AMSUS, 177(10), 1125-1130.

Jones, B., Cowan, D., Tomlinson, J., Robinson, J., Polly, D., \& Frykman, P. (1993). Epidemiology of injuries associated with physical training among young men in the Army. Medicine \& science in sports and exercise, 25(2), 197-203.

Liabsuetrakul, T., Prappre, T., Pairot, P., Oumudee, N., \& Islam, M. (2017). Development of a web-based epidemiological surveillance system with health system response for improving maternal and newborn health: field-testing in Thailand. Health informatics journal, 23(2), 109-123. https://doi.org/10.1177/1460458216628312

Luxton, D., Thomas, E., Chipps, J., Relova, R., Brown, D., McLay, R., Lee, T., Nakama, H., \& Smolenski, D. (2014). Caring letters for suicide prevention: Implementation of a multisite randomized clinical trial in the U. S. military and veteran affairs healthcare systems. Contemporary clinical trials, 37(2), 252-260.

Martínez, J. (2011). Aspectos psicológicos de la supervivencia en operaciones militares. Sanidad militar, 67(1), 43-48.

Ministerio del Trabajo (2013). II Encuesta Nacional de Condiciones de Seguridad y Salud en el Trabajo en el Sistema General de Riesgos Laborales. https://ccs.org.co/salaprensa/images/ Documentos/INFORME_EJECUTIVO_II\%20ENCSST.pdf

Mohammadi, F., Azma, K., Naseh, I., Emadifard, R., \& Etemadi, Y. (2013). Military exercises, knee and ankle joint position sense, and injury in male conscripts: a pilot study. Journal of athletic training, 48(6), 790-796.

Muniz, L., Sanches, M., Turi, B., \& Monteiro, H. (2017). Aptitud física y lesiones: 54 semanas de entrenamiento físico con la policía militar. Revista Brasileira de medicina do esporte, 23(2), 98-102.

Olmedilla, A., Ortega, E., Prieto, J., \& Blas, A. (2009). Percepción de los tenistas respecto a los factores que pueden provocar lesiones: diferencias entre federados y no federados. Cuadernos de psicología del deporte, 9(2), 7-18.

Organización Internacional del Trabajo. (1998). Principios directivos técnicos y éticos relativos a la vigilancia de la salud de los trabajadores. Oficina Internacional del Trabajo.

Pujol, M., \& Limón, E. (2013). Epidemiología general de las infecciones nosocomiales. Sistemas y programas de vigilancia. Enfermedades infecciosas y microbiología clínica, 31(2), 108-113.

Restrepo, C. (2013). Implementación de un modelo de vigilancia epidemiológica ocupacional para la intervención requerida de desórdenes musculo esqueléticos en trabajadores que utilizan computador en una institución de educación superior de ciudad de Popayán (Tesis de maestría). Universidad Autónoma de Occidente. 
Ríos, C., Castro, L., \& Melo, P. (2016). Lesiones derivadas del entrenamiento militar en los cadetes de $6^{\circ}$ nivel de la Escuela Militar José María Córdova. Revista movimiento científico, 10(1), 19-28.

Rodríguez, J., Valenzuela, J., Velasco, J., Castro, L., \& Melo, P. (2016). Caracterización de las lesiones derivadas del entrenamiento físico militar. Revista CUIDARTE, 7(1), 1219-26.

Spalj, S., Spalj, V., Ivankovic, L., \& Plancak, D. (2014). Oral health-related risk behaviours and attitudes among croatian adolescents - multiple logistic regression analysis. Collegium antropologicum, 38(1), 261-267. 